\title{
Predicting conjunction typicalities by component typicalities
}

\author{
GERT STORMS and PAUL DE BOECK \\ University of Leuven, Leuven, Belgium \\ JAMES A. HAMPTON \\ City University, London, England \\ and \\ IVEN VAN MECHELEN \\ University of Leuven, Leuven, Belgium
}

\begin{abstract}
In two studies, we investigated to what extent typicalities in conjunctive concepts phrased as relative clauses - such as pets that are also birds - can be predicted from simple functions of constituent typicalities and from extensions of such functions. In a first study, analyses of a large aggregated data set, based on seven different experiments, showed that a calibrated minimum rule model and some extensions of this model accounted for a very large part of the variance in the conjunction typicalities. The same models can also account for the so-called guppy effect. A psychological explanation is presented, which states that typicalities in contrast categories, like pets that are not birds and birds that are not pets, further improve the prediction of conjunction typicalities. This hypothesis is tested in a second study.
\end{abstract}

One of the central questions about conceptual combinations is what happens to constituent concepts that are combined into a complex concept. Different types of conceptual combinations have been distinguished: adjectivenoun combinations, such as sour wine (see, e.g., Murphy, 1988; Smith \& Osherson, 1984; Smith, Osherson, Rips, \& Keane, 1988), noun-noun combinations, such as pocket knife (Wisniewski, 1997; Wisniewski \& Gentner, 1991), and relative clause descriptions, such as pets that are also birds (Hampton, 1987, 1988; Storms, De Boeck, Van Mechelen, \& Geeraerts, 1993; Storms, Van Mechelen, \& De Boeck, 1994). In adjective-noun and nounnoun combinations, a considerable ambiguity may be present, in that a variety of relations can be implied. Ocean boat, ocean drive, and ocean book, for instance, all activate different relations between the head noun and the modifier of the compound (Murphy, 1988; Wisniewski, 1997). Relative clause descriptions of the form $X$ 's that are also $Y$ 's differ from noun-noun and adjective-noun combinations in that this sort of conceptual combination implies a clear type of relation between the complex concept and its constituents-namely, a which are also relation.

The authors thank Douglas Medin, Dedre Gentner, Edward Wisniewski, Lawrence Barsalou, and Lance Rips for discussions and comments on an earlier version of this manuscript, while the first author was visiting Northwestern University. He thanks Douglas Medin and Northwestern University for their hospitality. Correspondence concerning this article should be addressed to G. Storms, Department of Psychology, Tiensestraat 102, 3000 Leuven, Belgium (e-mail: gert. storms@psy.kuleuven.ac.be).
Although the majority of conceptual combinations are nonintersective (e.g., an expert repair is not the intersection of the classes of experts and repairs), our interest lies in the extent to which even explicitly intersective conceptual combinations may show systematic, unexpected patterns according to a simple logical conjunction. For this reason, we concentrate on relative clause descriptions of conjunctions, such as athletes that are also drug users. Anytime multiple classifications are made, a complex concept of the relative clause type is implied.

In the early eighties, Osherson and Smith (1981, 1982 ; Smith \& Osherson, 1984) argued that any hypothesis that combines typicalities ${ }^{1}$ of the constituent concepts according to a simple function, called simple functional hypotheses, must fail to predict typicalities for the conjunction. The argument, presented as part of a general critique of the prototype view on concept representation, has received considerable attention in the literature (for details, see, e.g., Cohen \& Murphy, 1984; Hampton, 1983, 1988; Jones, 1982; Kamp \& Partee, 1995; Lakoff, 1987; Thagard, 1983; Zadeh, 1982).

Osherson and Smith based their claim mainly on counterexamples and on intuitive examples. For example, they argued that, since wine gradually becomes vinegar if exposed to air, there must exist a sample $S$ that is an equally good example of wine and of vinegar. When considering the conjunctive concepts sour wine and sour vinegar, $S$ seems intuitively to be a better example of sour wine than of sour vinegar. This, however, cannot be explained with any simple functional hypothesis, since the typicality of $S$ for sour is invariant in the two combinations and $S$ was chosen precisely to have an equal typicality in wine and 
Table 1

Number of Participants, Conjunctions, and Items per Conjunction in the Seven Experiments

\begin{tabular}{|c|c|c|c|}
\hline \multirow[b]{2}{*}{ Experiment } & \multicolumn{3}{|c|}{ Number } \\
\hline & Participants & Conjunctions & $\begin{array}{l}\text { Items per } \\
\text { Conjunction }\end{array}$ \\
\hline Hampton (1988), Experiment 2 & 36 & 1 & 43 \\
\hline Hampton (1988), Experiment 3 & 55 & 1 & 36 \\
\hline Hampton (1988), Experiment 4 & 40 & 6 & 16 \\
\hline Hampton (1997) & 74 & 6 & $17-20$ \\
\hline Hampton (unpublished) & 40 & 6 & 32 \\
\hline $\begin{array}{l}\text { Storms, De Boeck, Van Mechelen, } \\
\text { and Geeraerts (1993) }\end{array}$ & 40 & 5 & 40 \\
\hline $\begin{array}{l}\text { Storms, De Boeck, Van Mechelen, } \\
\text { and Ruts (1996) }\end{array}$ & 125 & 50 & 30 \\
\hline
\end{tabular}

in vinegar. In some of their publications, however, Smith and Osherson (1984; Smith, Osherson, Rips, \& Keane, 1988) also presented experimental data to back up their claims against the existence of any simple functional hypothesis and against one function in particular-namely, the minimum rule model proposed by Zadeh $(1965,1982)$, which states that the typicality of an item as a conjunction of two concepts (conjunction typicality) equals the minimum of the typicalities of the two constituents. Other authors, however, have shown that a simple function can do fairly well in predicting the typicality of a conjunction. For example, Hampton (1988) found that typicalities of a conjunction were correlated highly $(R$ between .90 and .97 ) with a linear combination of typicalities of the corresponding constituents, for conjunctions phrased as relative clauses, such as pets that are also birds.

In a first study, a large body of empirical data will be analyzed. It will be shown that the typicalities of the conjunction can be explained, to a surprisingly large extent, by elemental typicalities and their relations. These results lead to a psychological explanation that states that typicalities for the constituents together with typicalities of contrast categories further improve the prediction of conjunction typicalities. In a second study, this contrast category hypothesis is tested.

\section{STUDY 1}

In the first study, we wanted to obtain a general impression of how the typicalities of conjunctions vary as a function of typicalities of both constituents. Therefore, data from seven different experiments were aggregated into one large data set, to be analyzed by different models with varying complexity but all meeting the requirements of simple functional hypotheses, as defined by Osherson and Smith (1982, p. 304) - that is, a function $f$, such that, for all conjunctive concepts $A \& B$,

$$
(\forall x \in D) c_{A \& B}(x)=f\left[c_{A}(x), c_{B}(x)\right],
$$

where $D$ is the domain of discourse, $c_{A}, c_{B}$, and $c_{A \& B}$ are characteristic functions that reflect typicality and that map $D$ into $[0,1]$. Before describing the applied simple functional hypotheses in detail, we will first focus on the aggregated data set.

We aimed to gather a large data set with aggregated data from as many experiments as possible. In the literature, five publications describe experiments in which typicalities in relative clause descriptions of conjunctions have been studied (Chater, Lyon, \& Myers, 1990; Hampton, 1988, 1997; Storms et al., 1993; Storms, De Boeck, Van Mechelen, \& Ruts, 1996). Unfortunately, the data from Chater et al. are no longer available ( $\mathrm{N}$. Chater, personal communication, September 1995). The data used in the present study come from the four remaining publications, plus one unpublished study, and are taken from seven different experiments. In all the experiments, rated typicalities for different item sets were gathered for conjunctive concepts phrased as relative clause descriptions (phrased in both orders: $A$ 's that are also $B$ 's and $B$ 's that are also $A$ ' $s$ ), as well as for the constituents ( $A$ 's and $B$ 's). In each of the studies, 7-point rating scales ranging from -3 (unrelated) to +3 (very typical) were used, with zero marking the category boundary (see Hampton, 1988).

Table 1 shows details about the number of participants and the number of conjunctions studied in the seven experiments. For all the experiments except Storms et al. (1996), three types of items were investigated: members of the conjunction category, members of one constituent but not of the other, and items that were not members of either of the constituent categories. The latter type was not included in Storms et al. (1996).

The reliability of the typicality ratings was estimated by applying the Spearman-Brown formula to the splithalf correlation, after randomly dividing each of the participant groups who rated the typicality of a list of items in relation to the same category label into two groups of equal size. A mean estimated reliability of .95 was obtained for typicality ratings for the constituent concepts, and a mean estimate of .90 for the conjunctive concepts. Note that this implies that $90 \%$ of the variance in the conjunction ratings is true variance (i.e., the rest is error variance; any model can account for maximally $90 \%$ of 
the observed variance). For more details about materials, procedures, and data, the reader is referred to the original publications.

Aggregation over experiments and conjunctions resulted in a data set consisting of 2,187 items, with variables referring to mean ratings across participants for each constituent category and for the conjunction (averaged across the two different phrasings of the conjunctions). (From Experiments 1-6,687 items were taken, including items belonging to neither constituent. The remaining 1,500 items were from Experiment 7 and did not include the latter type of items.) In sum, the data were gathered from more than 490 participants, all of them students, 190 of whom were native Dutch speakers participating in experiments conducted by Storms and his colleagues, who used Dutch materials. The remaining subjects were native English speakers (British and American) who participated in Hampton's experiments, which were entirely in English.

\section{Simple Functional Hypotheses and Results}

Osherson and Smith $(1981,1982,1997)$ argued that the simple minimum rule is often violated for typical examples of the conjunctive category, such examples often being more typical for the conjunction than for both constituents. Osherson and Smith stated that a guppy is intuitively a more typical example of the conjunctive category pet fish than it is of either pets or fish. Yet, an empirical test of this claim (Storms, De Boeck, Van Mechelen, \& Ruts, 1998) showed that the case of guppy as a pet fish is not, in fact, a good example of this so-called guppy effect, but that other and better examples can be found to substantiate the claim. Smith and Osherson (1984; Smith et al., 1988) also presented ample evidence that, in adjective-noun compounds, many examples are more typical for the conjunction than for the noun concept. This phenomenon, called the conjunction effect, also characterizes relative clause descriptions of conjunctions (Storms, Ruts, \& Vandenbroucke, 1998). Both the guppy effect and the conjunction effect are incompatible with the simple minimum rule. One may wonder, however, whether the incompatibility could be removed by changing the minimum rule slightly. There are two straightforward extensions of the minimum rule that we want to consider: First, one can simply allow the minimum to be weighted (hereinafter called the weighted minimum rule model), and second, one can allow a simple calibration of the response scale (hereinafter called the calibrated minimum rule model) in order to distribute typicality ratings more equally across the scale (Jones, 1982; Kamp \& Partee, 1995; for a related discussion concerning overextensions, see Huttenlocher \& Hedges, 1994).

The weighted minimum rule model can be evaluated with a regression model through the origin, with the minimum constituent typicality as predictor (i.e., no additive constant is allowed). When fit to the data, this simple model accounted for a surprising $70.8 \%$ of the variance in the conjunction typicalities $(r=.842) .^{2}$

A calibration shift can be incorporated into the weighted minimum rule model by including a constant in the regression function. When this calibrated minimum rule model was fit to the aggregated data set, as a linear model with two parameters (for the slope and the intercept), $80.1 \%$ of the variance in the typicalities of the conjunction could be accounted for $(r=.895)$. The minimum constituent typicality was a highly significant predictor $(p<.0001)$, and the added constant was .59 , meaning that the zero point of the typicality rating scale (ranging from -3 to +3 ) had shifted more than half a unit. The value of the additive constant was significantly larger than zero $(p=.0001)$, a finding that provides evidence against the simple minimum rule model. In other words, items were judged to be more typical for the conjunction than would be predicted by the simple minimum rule model. Note that the results described above were obtained with a model that included only two free parameters (the additive constant and the weight for the minimum predictor). In other words, the model estimated no conjunctionspecific parameters.

Despite the surprisingly good prediction, some results reported in the literature suggest that the calibrated minimum rule model could still be improved. In particular, Hampton $(1987,1988)$ found that, in conjunctions, one of the constituents may have a (significantly) larger influence on the conjunction than does the other constituent. For instance, when predicting typicality ratings for sports that are also games or games that are also sports on the basis of the ratings for sports and games, the sports ratings had a higher impact on the conjunction ratings than did the games ratings. This so-called dominance effect was replicated by Storms et al. (1993; Storms et al., 1996). The calibrated minimum rule model can never capture that part of the variance that is caused by the dominance effect. However, the model can easily be extended so as to incorporate a differential weight for each of the two constituents. Note that the minimum of two numbers equals 0.5 times their (unweighted) sum minus 0.5 times the absolute difference between them. When the sum is decomposed into its two constituents, a model can be fit with three predictors: typicalities for both of the constituents and their absolute difference. This model extends the calibrated minimum rule model by allowing the two constituents to have different weights and by also allowing the weight of the absolute difference to vary freely. We will call this model the calibrated minimum rule model adjusted for dominance. This model will only succeed in capturing (part of) the dominance effect if one of the two predictor variables refers to the dominant constituent of every conjunctive combination. Therefore, for every concept pair, the dominant constituent was determined empirically (whichever of the two constituents had the highest correlation with the conjunction), and the dominant 
and nondominant constituents were labeled $A$ and $B$, respectively. (Note that the discrimination between the dominant and the nondominant constituents is done a posteriori. This model, therefore, does not meet the simple functional hypothesis requirements.) Fitting the minimum rule model adjusted for dominance explained $82.7 \%$ of the variance in the conjunction typicalities (to be compared with the $80.1 \%$ for the calibrated minimum rule model). All three predictor variables (dominant and nondominant constituent plus the absolute difference) entered significantly into the regression equation $(p<.0001)$. This model used two more free parameters than did the calibrated minimum rule model, and the gain in predictive value was significant $[F(2,2183)=168.9, p<.01]$. The dominance effect did not explain very much additional variance, although it is a well-established phenomenon (Hampton, 1987, 1988; Storms et al., 1996), because the model only includes an average dominance effect, since no conjunction-specific parameters are fit. (Storms et al., 1996, showed that part of the dominance effect is accounted for by a difference in variance of the typicalities for the two constituents. This part of the dominance effect cannot be captured by any simple functional hypothesis, because the size of the variance of the typicalities in the two constituents may differ across the different concept pairs.) The additive constant, although still significantly different from zero $(p=.0015)$, was much smaller than in the calibrated minimum rule model $(0.12)$.

The three models presented above can also be used to predict items that show the so-called guppy effect (i.e., items for which the rated conjunction typicality exceeds the rated typicality of both constituents). All the items for which the predicted conjunction typicality is larger than the rated constituent typicalities are "predicted" guppy items. The empirical data set contained 174 guppy items. The weighted minimum rule model, the calibrated minimum rule model, and the calibrated minimum rule model adapted for dominance yielded 41, 277, and 155 "predicted" guppy items, respectively. Furthermore, we checked whether the number of 174 empirically observed guppy items is representative of what can be expected if our study were replicated under the assumption that each of the three models (assuming a normally distributed error component) was true. For this, we made use of a Monte Carlo significance testing procedure (Efron \& Tibshirani, 1993; Hope, 1968). ${ }^{3}$ It appears that, for all three models, the empirically observed number is not higher than the replicated numbers of guppy items ( $p=$ $.45, .84$, and .51 , respectively).

The data from the aggregated data set can be visualized in a three-dimensional space, with typicality ratings for both constituents defining the two dimensions of a ground plane and with the conjunction typicalities defining the third dimension (height). For the three models discussed so far, the surface representing the predicted conjunction typicalities then looks like two half planes that meet along the diagonal rising from the low-low corner to the high-high corner. We considered to what extent the shape of the surface could be adapted so as better to fit the conjunction typicalities without making the surface too irregular. To answer this question, a bidimensional cubic spline function was fit to the aggregated data set, using the FITPACK algorithm of Dierckx (1981, 1993). A spline function is a very flexible device to perform (bidimensional) curve fitting; it does not force the predicted conjunction typicalities to take the form of two planes. ${ }^{4}$

Figure 1 shows the contour plot of the cubic spline function that accounted for approximately $84 \%$ of the variance in the conjunction typicalities. This percentage may be considered to be a reasonable maximum one can attain with a simple function, especially taking into account the reliability of the rated typicalities and the fact that a single general function has to fit all 75 concept pairs, with no conjunction-specific parameters. The contour plot of the spline function showed that the typicality ratings for the conjunction increased generally in a direction going from the low-low corner to the highhigh corner. This corresponds to the (significant) positive regression coefficient of the minimum predictor in the calibrated minimum rule model and to the (significant) positive regression coefficients of the typicalities of both constituents in the calibrated minimum rule model adjusted for dominance. The curved shape of the height lines in Figure 1, however, shows that the increasing surface differed considerably from a plane and that there was a ridge more or less along the diagonal, going from the low-low to the high-high corner. This ridge can be seen clearly in Figure 2, where the cross section at the diagonal going from the high-low to the low-high corner is shown. Such a ridge was also predicted by both previous models, owing to the inclusion of the absolute difference as (part of) a predictor. Thus, both the calibrated and the adjusted model, as well as the spline function, reflect that, for any given sum of typicalities for the two constituents, the more equal the typicalities for the two constituents are, the higher will be the typicality of the conjunction. Note also that, owing to the dominance effect, the ridge in the spline solution is not located exactly along the diagonal but is shifted somewhat to the right (see Figures 1 and 2). Besides, the decrease from the center toward the low-high and the high-low corners is not linear, as is predicted by the calibrated and the adjusted minimum rule models, but gets stronger as the difference between the typicalities of the two constituents increases.

In sum, the bidimensional spline function, although it did not impose any serious restrictions on the solution, did show the same two general characteristics that were present in the calibrated minimum rule model and the minimum rule model adjusted for dominance: (1) general increasing conjunction typicalities along the diagonal from the low-low to the high-high corners and (2) an inverse U-shaped pattern along the diagonal from the lowhigh to the high-low corners. The increment in explained variance relative to the calibrated minimum rule model adjusted for dominance was only just over $1 \%$. 


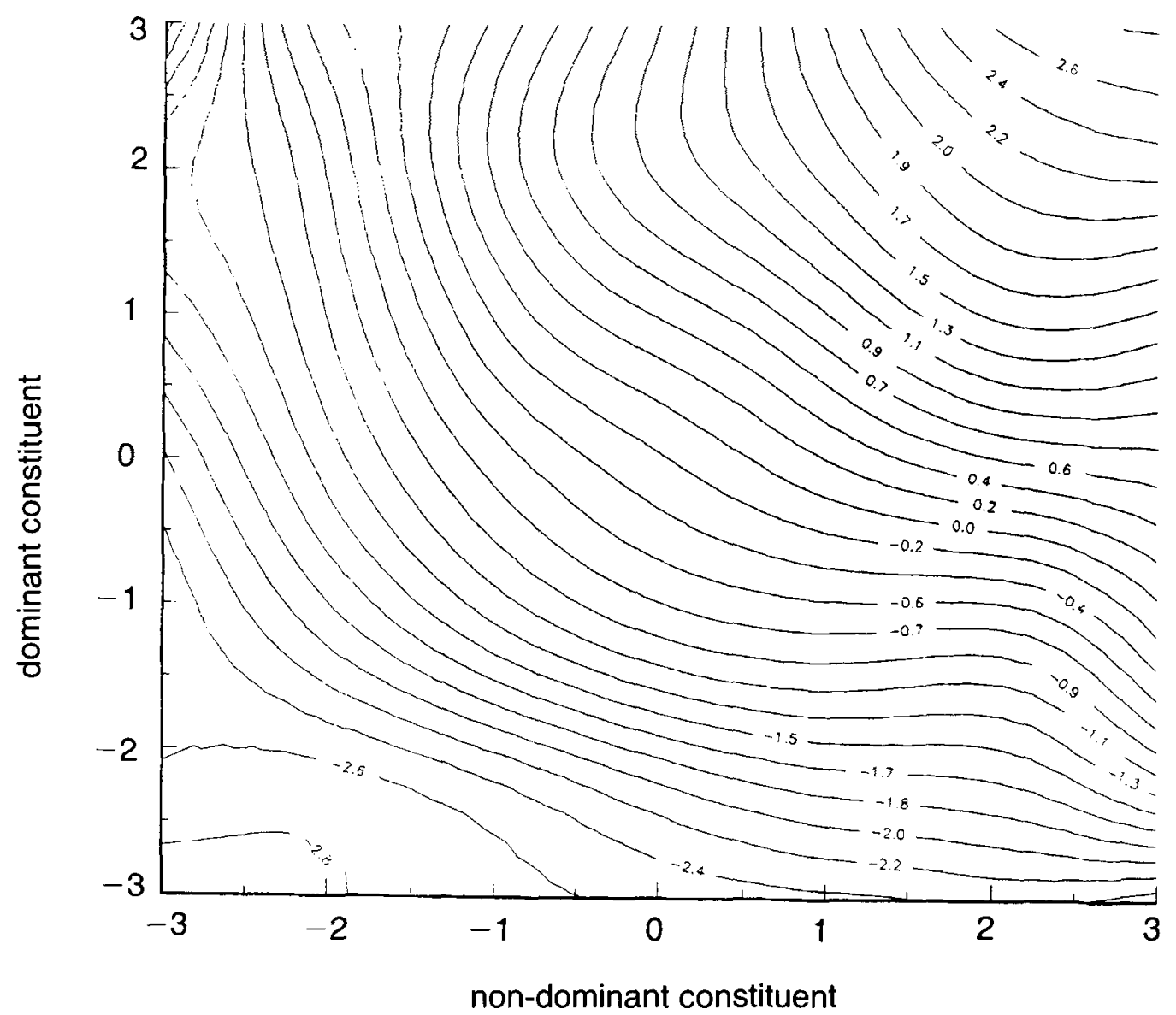

Figure 1. Contour plot of the bidimensional splines solution for the conjunction typicalities.

A serious drawback of the models fit so far, although they were successful in a predictive sense, is that they are just formulas without a cognitive theory. A simple cognitive explanation for the bending down of the surface toward the high-low and the low-high corners is that, for conjunctions, as for simple concepts, contrast categories play a role. The most natural contrasts for the conjunction $A \& B$ are $A \&$ not $B$ and $B \&$ not $A$. Typicalities for these contrast categories can be expected to increase, the closer an item is situated to the low-high or the high-low corner. Adding typicalities for these contrast categories to the regression model may, therefore, improve the prediction of conjunction typicalities. Also, a closer look at the crosssection of the spline function along the low-high to highlow diagonal (Figure 2) showed that the bending down along this diagonal is stronger as one comes closer to the these corners, suggesting that the influence of the contrast categories might increase, the more typical an item is for a constituent. Such a phenomenon could be modeled by adding two interaction terms as predictor variables: the product of the typicalities of $A$ and of $A$ 's that are not $B$ 's, and the product of $B$ and of $B$ 's that are not $A$ 's. It does make sense that a composite contrast category is more relevant, the worse its positive constituent applies. The contrast hypothesis and the interaction term hypothesis will be tested in an explicit way in Study 2 .

\section{STUDY 2}

To evaluate the contrast category and the interaction term hypotheses, a new experiment was conducted. In this experiment, typicality ratings were gathered for the constituent concepts ( $A$ 's and $B$ 's), the conjunctions ( $A$ 's that are also $B$ 's and $B$ s that are also $A$ 's), and for two contrast categories ( $A$ 's that are not $B$ 's and $B$ 's that are not $A$ 's).

\section{Method}

Participants. Eighty first-year psychology students at Leuven University participated for course credit.

Materials. Nine different conjunctions, taken randomly from Storms et al. (1996), were selected: causes of death-diseases, coats-evening wear, fruits-desserts, birds-pets, machines-vehicles, sports-games, writing implements-office equipment, footwear sports equipment, and furniture-household appliances. For each conjunction, a set of 40 items was selected, consisting of 10 membets of the conjunction, 10 members of each constituent category $A$ and $B$ that were not members of the other constituent category, and 10 related items that were not members of either of the constituents but of a superset category $S$ (e.g., animals for the conjunction of pets and birds). 


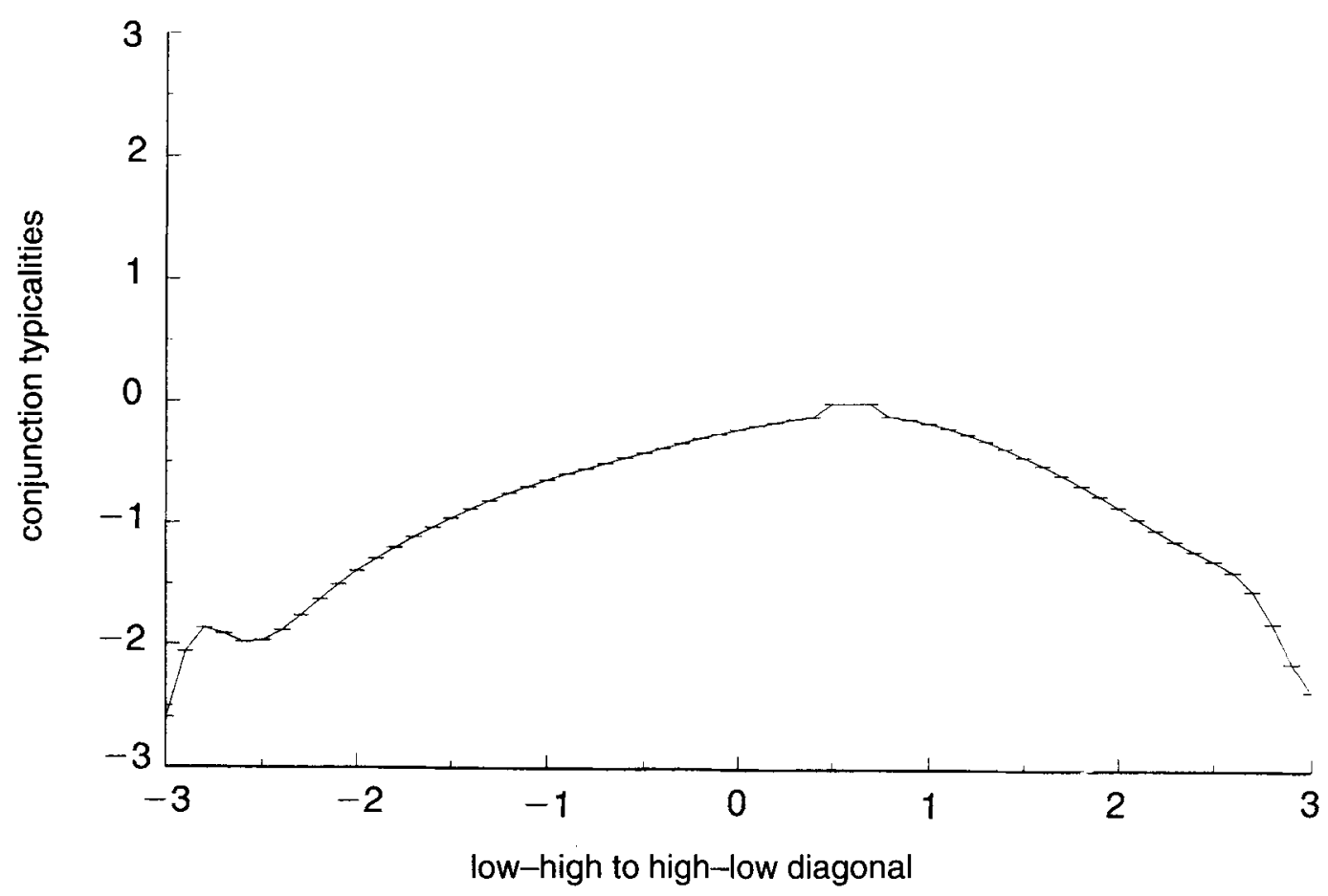

Figure 2. Cross section of the splines solution along the diagonal going from the low-high to the high-low corners.

Procedure. Ratings were made for the following categories: $A$ 's, $B$ 's, $A$ 's that are also $B$ 's, $B$ 's that are also $A$ 's, $A$ 's that are not $B$ 's, $B$ 's that are not $A$ 's, and $S$ 's that are neither $A$ 's nor $B$ 's. Since a definition of not $A$ and not $B$ cannot be given without referring to a superset category $S$, we decided, as a way of balancing the materials, to add the superset category in the rating instructions for all categories (e.g., animals that are both pets and birds, etc.). The items of each conjunction were rated by 10 different participants, and every participant rated the items of all nine conjunctions. The order of the nine conjunctions was balanced over the different participants.

\section{Results}

The reliability of the typicality ratings was estimated by applying the Spearman-Brown formula to the split-half correlation, after randomly dividing each of the participant groups who rated the typicality of a list of items in relation to the same category label into two groups of equal size. Mean estimated reliabilities equaled $.98, .94$, and .96 , for typicalities of constituent categories, of conjunctive categories, and of contrast categories, respectively.

The following models were fit jointly, for the nine different conjunctions, to the data averaged over subjects. (All of the models were also fit by including the contrast notion $S$ that are not $A$ and not $B$, but since it was never a significant predictor, these results are not reported.) The weighted minimum rule model accounted for $80.7 \%$ of the variance in the conjunction typicalities. The calibrated minimum rule model and the model adjusted for dominance explained, respectively, $84.6 \%$ and $88.9 \%$ of the variance [incremental $F(2,356)=68.9, p<.01]$. All the predictor variables entered significantly in all models $(p<.0001)$. The simple contrast model and the inter- action contrast model accounted for $86.5 \%$ and $89.9 \%$ of the variance, respectively [incremental $F(2,353)=50.9$, $p<.01]$. In the simple contrast model, only the typicalities of one of the two contrast categories ( $A$ 's that are not $B s)$ entered in significantly $(p<.0001)$. In the interaction contrast model, the typicalities of both contrast categories reached significance, as well as the interaction of $A$ and $A$ 's that are not $B$ 's. The remaining interaction (of $B$ and $B$ 's that are not $A$ 's) was not significant.

A linear regression model with the absolute difference as a criterion and the typicalities of the contrast categories as predictors, accounted for $77.5 \%$ of the variance in the absolute difference. These results indicated that the absolute difference and the typicalities of the contrast categories did not carry exactly the same information. Adding the absolute difference to the interaction contrast model accounted for $90.4 \%$ of the variance of the conjunction typicalities. This is an increase of $0.5 \%$ of explained variance, which was nevertheless significant [incremental $F(1,352)=18.3, p<.01]$.

Regarding the guppy effect, Monte Carlo significancetesting procedures again showed that the observed number of guppy items was not significantly different from what would be expected from the three models under consideration.

For the data of Hampton's (1997) negation study, which were also included in the aggregated data set used in our first study, ratings of the contrast categories were also available. Analyses similar to those described above were carried out on these data. The results of these analyses were completely in line with the results of Study 2 . 


\section{DISCUSSION AND CONCLUSION}

Our studies have shown that Osherson and Smith's $(1981,1982)$ dismissal of the simple minimum rule was warranted, in the sense that a weighting of the minimum constituent typicality and an additive constant in the model significantly improves predictability of conjunctions on the basis of their constituents. However, when fit to a very large data set obtained from conjunctions phrased as relative clause descriptions, a weighted and a calibrated minimum rule model account for a very substantial proportion of the variance in the conjunction typicalities. This "simple functional hypothesis" is shown to be improved on by additionally taking into account that one constituent may dominate the other. The maximum percentage of variance that can be explained with a simple model derivable from the constituent typicalities turns out to be $84 \%$, as is shown by the obtained spline function.

As an explanation of an important feature of the spline function, we hypothesized that contrast categories of the conjunction play a role. New data gathered in Study 2, as well as data available from Hampton (1997), provided clear evidence for the importance of two negation contrast categories for the conjunction. Moreover, it was shown that the effect of the categories $A s$ that are not $B\}$ and $B$ 's that are not $A$ 's is stronger, the more typical an item is for $A$ and for $B$, respectively.

The contrast effect provides a cognitive interpretation for why the absolute difference contributes to the prediction in both the simple calibrated minimum rule model and the calibrated minimum rule model adjusted for dominance. The importance of contrast categories has been advocated in many theories and models of simple semantic concepts. In prototype theory, for instance (Rosch, 1975, 1978; Rosch \& Mervis, 1975), items are considered to be more typical exemplars of a category, the more attributes they have in common with other exemplars of the category and the fewer attributes they have in common with exemplars of contrast categories. Also, many exemplar-based models of category learning assume that the probability of classifying an item in a category is an increasing function of its similarity toward other learned exemplars of the category and a decreasing function of its similarity toward learned exemplars of contrast categories (see, e.g., Medin \& Schaffer, 1978; Nosofsky, 1984). (For other examples of the importance of contrast categories, see also Malt \& Johnson, 1992, and Markman \& Wisniewski, 1997.) By incorporating typicalities of contrast categories, we obtained a model for conjunction typicalities that is no longer just a simple prediction formula based on the constituents. It comprises terms inspired by a cognitive theory.

\section{REFERENCES}

Chater, N., Lyon, K., \& Myers, T. (1990). Why are conjunctive categories extended? Journal of Experimental Psychology: Learning. Memory, \& Cognition, 16, 497-508.
Cohen, B., \& Murphy, G. L. (1984). Models of concepts. Cognitive Science, 8, 27-58.

DEBoor, C. (1978). A practical guide to splines. New York: SpringerVerlag.

DIERCKX, P. (1981). An algorithm for surface fitting with spline functions. IMAJ: Numerical Analysis, 1, 267-283.

DiERCKX, P. (1993). Curve and surface fitting with splines (Oxford Monographs on Numerical Analysis). Oxford: Oxford University Press, Clarendon Press.

Efron, B., \& Tibshirani, R. J. (1993). An introduction to the bootstrap. New York: Chapman \& Hall.

HAMPTON, J. A. (1983). A composite prototype model of conceptual conjunction. Unpublished manuscript, City University, London.

HAMPTON, J. A. (1987). Inheritance of attributes in natural concept conjunctions. Memory \& Cognition, 15, 55-71.

HAMPTON, J. A. (1988). Overextension of conjunctive concepts: Evidence for a unitary model of concept typicality and class inclusion. Journal of Experimental Psychology: Learning, Memory, \& Cognition, 14, 12-32.

Hampton, J. A. (1997). Conceptual combination: Conjunction and negation of natural concepts. Memory \& Cognition, 25, 888-909.

HOPE, A. C. A. (1968). A simplified Monte Carlo significance test procedure. Journal of the Royal Statistical Society: Series B, 30, 582-598.

Huttenlocher, J., \& HedGes, L. V. (1994). Combining graded categories: Membership and typicalities. Psychological Review, 101, $157-165$.

JONES, G. V. (1982). Stacks not fuzzy sets: An ordinal basis for prototype theory of concepts. Cognition, 12, 281-290.

Kamp, H., \& Partee, B. (1995). Prototype theory and compositionality. Cognition, 57, 129-191.

LAKOFF, G. (1987). Women, fire, and dangerous things. Chicago: University of Chicago Press.

Malt, B. C., \& JohnSon, E. C. (1992). Do artifact concepts have cores? Journal of Memory \& Language, 31, 195-217.

Markman, A. B., \& WisniewSKI, E. J. (1997). Similar and different: The differentiation of basic-level categories. Journal of Experimental Psychology: Learning, Memory, \& Cognition, 23, 1-17.

Medin, D. L., \& Schaffer, M. M. (1978). Context theory of classification learning. Psychological Review, 85, 207-238.

Murphy, G. L. (1988). Comprehending complex concepts. Cognitive Science, 12, 529-562.

NosofsKy, R. M. (1984). Choice, similarity and the context model of classification. Journal of Experimental Psychology: Learning, Memory, \& Cognition, 10, 104-114.

OSHERSON, D. N., \& SMITH, E. E. (1981). On the adequacy of prototype theory as a theory of concepts. Cognition, 11,35-58.

Osherson, D. N., \& SMith, E. E. (1982). Gradedness and conceptual conjunction. Cognition, 12, 299-318.

OsherSON, D. N., \& SMITh, E. E. (1997). On typicality and vagueness. Cognition, 64, 189-206.

Rosch, E. (1975). Cognitive representations of semantic categories. Journal of Experimental Psychology: General, 104, 192-233.

Rosch, E. (1978). Principles of categorization. In E. Rosch \& B. B. Lloyd (Eds.), Cognition and categorization (pp. 27-48). Hillsdale, $\mathrm{NJ}$ : Erlbaum

Rosch, E., \& Mervis, C. B. (1975). Family resemblances: Studies in the internal structure of categories. Cognitive Psychology, 7, 573-605.

Smith, E. E., \& Osherson, D. N. (1984). Conceptual combination with prototype concepts. Cognitive Science, 8, 337-361.

Smith, E. E., Osherson, D. N., Rips, L. J., \& Keane, M. (1988). Combining prototypes: A selective modification model. Cognitive Science, 12, 485-527.

Storms, G., De Boeck, P., Van Mechelen, I., \& Geeraerts, D. (1993). Dominance and noncommutativity effects in concept conjunctions: Extensional or intensional basis? Memory \& Cognition, 21, 752-762.

Storms, G., De Boeck, P., Van Mechelen, I., \& Ruts, W. (1996). The dominance effect in concept conjunctions: Generality and interaction aspects. Journal of Experimental Psychology: Learning, Memory, \& Cognition, 22, 1-15. 
Storms, G., De Boeck, P., Van Mechelen, I., \& Ruts, W. (1998). Not guppies, nor goldfish, but tumble dryers, Noriega, Jesse Jackson, panties, car crashes, bird books, and Stevie Wonder. Memory \& Cognition, 26, 143-145.

Storms, G., Ruts, W., \& Vandenbroucke, A. (1998). Dominance, overextensions, and the conjunction effect in different syntactic phrasings of concept conjunctions. European Journal of Cognitive Psychology, 10, 337-372.

Storms, G., Van Mechelen, I., \& De Boeck, P. (1994). Structural analysis of the intension and extension of semantic concepts. European Journal of Cognitive Psychology, 6, 43-75.

Thagard, T. (1983, June). Conceptual combination: A frame-based theory. Paper presented at the Society for Philosophy and Psychology, Wellesley, MA.

WISNIEWSKI, E. J. (1997). When concepts combine. Psychonomic Bulletin \& Review, 4, 167-183.

WISNIEWSKI, E. J., \& GENTNER, D. (1991). On the combinatorial semantics of noun pairs: Minor and major adjustments to meaning. In G. B. Simpson (Ed.), Understanding word and sentence (pp. 241-284). Amsterdam: North-Holland.

ZADEH, L. (1965). Fuzzy sets. Information \& Control, 8, 338-353.

ZADEH, L. (1982). A note on prototype theory and fuzzy sets. Cognition, 12, 291-297.

\section{NOTES}

1. Throughout this paper, we use the term typicality to refer to both the goodness of example of a category member and gradedness, or the degree to which borderline examples or nonmembers belong in the cat- egory. Although Osherson and Smith (1997) have argued that these two phenomena can be distinguished, our experimental procedure asked subjects to combine them into a single scale.

2. We thank Fintan Costello for this suggestion.

3. In particular, 100 Monte Carlo samples with the same size as the empirically observed data were drawn from each model, with the parameters set at their estimated values for the observed data and with the constituent typicalities kept at their observed values. For each sample, the number of guppy items was calculated and compared with the empirically observed number.

4. The algorithm divides the ground plane constituted by the typicalities of both constituents into a number of rectangles by cutting it horizontally and vertically at different knots. In each of the rectangles, a cubic polynomial is fit to predict conjunction typicalities from the typicality of the two constituents, in such a way that, on all points on an edge of two bordering rectangles, the values of the first, second, and third derivative are continuous, making the overall surface of the spline function smooth over the whole domain. (For a general introduction to spline functions, see deBoor, 1978.) The FITPACK algorithm selects the location of the knots optimally according to a least-squares principle. The number of selected knots depends on the precision with which the spline function is required to approximate the data. Different levels of precision were tried, to find a solution that was close enough to the data to get an overview of the surface but that did not use too many free parameters.

(Manuscript received April 30, 1998; revision accepted for publication December 20, 1998.)

\title{
Nominations for the Editorship of Memory \& Cognition
}

Nominations are solicited for the editorship of Memory \& Cognition. The term of the present editor, Morton Ann Gernsbacher, expires at the end of 2001. The new editor will begin an official 5-year term on January 1, 2002, and will begin to receive manuscripts early in January 200 I. The Publications Committee of the Psychonomic Society expects to appoint the new editor by December 2000.

Nominations (including self-nominations) should be submitted by May 1, 2000, to:

\author{
Geoff Loftus \\ Chair, Memory \& Cognition Search Committee \\ Department of Psychology, Box 351525 \\ University of Washington \\ Seattle, WA 98195
}

